\title{
Enhanced radiosensitizing by sodium glycididazole in a recurrent esophageal carcinoma tumor model
}

\author{
Peipei Wu ${ }^{1,2, *}$, Jing Liu ${ }^{1, *}$, Xiaorong Sun ${ }^{3}, X$ iaolin $\mathbf{L i}^{1}$, Ligang Xing ${ }^{1}$ and Jinming $\mathbf{Y u}^{1}$ \\ ${ }^{1}$ Department of Radiation Oncology, Shandong Key Laboratory of Radiation Oncology, Shandong Cancer Hospital Affiliated to \\ Shandong University, Shandong Academic of Medicine Science, Jinan 250117, Shandong, China \\ ${ }^{2}$ Department of Oncology, Jining No.1 People's Hospital, Jining 272011, Shandong, China \\ ${ }^{3}$ Department of Radiology, Shandong Key Laboratory of Radiation Oncology, Shandong Cancer Hospital Affiliated to Shandong \\ University, Shandong Academic of Medicine Science, Jinan 250117, Shandong, China \\ *These authors have contributed equally to this work \\ Correspondence to: Ligang Xing, email: xinglg@medmail.com.cn \\ Keywords: esophageal cancer, sodium glycididazole, radiosensitizer, tumor bed effect, hypoxia \\ Received: December 15, $2016 \quad$ Accepted: June 05, $2017 \quad$ Published: July 10, 2017 \\ Copyright: Wu et al. This is an open-access article distributed under the terms of the Creative Commons Attribution License 3.0 \\ (CC BY 3.0), which permits unrestricted use, distribution, and reproduction in any medium, provided the original author and source \\ are credited.
}

\section{ABSTRACT}

Re-irradiation is challenging for esophageal cancer patients with local-regional recurrence after initial radiotherapy. The purpose of this study is to establish a recurrent esophageal tumor model and investigate radiosensitizing effects of sodium glycididazole ( $\mathrm{CMNa}$ ). Tumor models were established by pre-irradiation ( $0 \mathrm{~Gy}, 10 \mathrm{~Gy}$ or $20 \mathrm{~Gy}$ ) to the right hind leg of the nude mice 24 hours before tumor transplantation (ECA109 human esophageal carcinoma cells). Tumor growth curves were analyzed. Hypoxic microenvironment was exhibited in tumor frozen slides stained for pimonidazole, Hoechst 33342, hematoxylin-eosin and CD34. Mice bearing primary ( $0 \mathrm{~Gy}$ pre-irradiation) and recurrent (10 Gy pre-irradiation) tumors were randomized into control (no treatment), radiation ( $30 \mathrm{~Gy}$ in 3 weekly fractionations), or radiation combined with $\mathrm{CMNa}(1 \mathrm{mmol} / \mathrm{kg}$ i.p. injected $60 \mathrm{~min}$ before radiation) respectively. The data showed tumors from $10 \mathrm{~Gy}$ and $20 \mathrm{~Gy}$ pre-irradiated sites grew significantly slower than those in the 0 Gy pre-irradiated group. The recurrent xenograft tumors showed increased necrotic fractions, decreased micro-vascular density, increased pimonidazole-positive fraction, and decreased Hoechst-positive fraction. In the primary xenograft tumors, $\mathrm{CMNa}$ adding to radiation did not lead to significant tumor growth delay than radiation alone. However, for the recurrent tumor model, the growth rate was remarkably reduced as CMNa combined with radiation as comparison with radiation alone. In conclusion, the recurrent esophageal xenograft model with tumor bed effect was successfully established characterized by slow growth, increased hypoxia fraction and decreased blood flow. Significant radiosensitization by CMNa was demonstrated in the recurrent model.

\section{INTRODUCTION}

Radiotherapy is the mainstay in management of esophageal carcinoma [1, 2]. However, local-regional recurrence after radiotherapy was about $40 \sim 50 \%$ despite of advancing radiation techniques and advanced chemotherapy/targeted therapy [3, 4]. Salvage reirradiation with/without chemotherapy is an important choice, but it is challenging because normal tissue already got high-dose irradiation during first setting of treatment. More importantly, the radiobiological characteristics of recurrent tumor after radiotherapy are different from the primary tumors $[5,6]$.

To elucidate its biology, serial xenograft models for recurrent tumors were established. Tumor transplanted into pre-irradiated tissue has been frequently used as 
recurrent xenograft tumor models [5-8]. Recurrent tumors were often found to be resistant to re-irradiation, resulted in poor disease control and limited survival $[6,9,10]$. This could be due to the condition known as "tumor bed effect" (TBE), which showed tumors in pre-irradiated beds commonly characterized by reduced blood perfusion, extensive necrosis, and elevated hypoxic fractions. Existed study [9] showed that TBE appeared at single 5-30 Gy irradiation or 40-60 Gy total dose in a conventional fractionation mode dependent on different tumor types. However, recurrent tumor model in esophageal cancer was not well-documented.

As the reported higher hypoxia fraction in the recurrent tumors, it is possible to improve the radiation effect using hypoxia radiosensitizer. However, few related studies were reported. Sodium glycididazole (C18H22N7NaO10.3H2O), abbreviated CMNa, is a new nitroimidazole compound independently developed in China. Experimental and clinical studies [11-20] reported significant radiosensitizing effects of $\mathrm{CMNa}$ in solid tumors without adverse influences on normal tissues. The aims of present preclinical study are to: (1) establish a recurrent esophageal tumor model, (2) define the hypoxic microenvironment of the established recurrent tumor model, and (3) investigate the radiosensitizing effects of $\mathrm{CMNa}$ in the recurrent tumor model.

\section{RESULTS}

\section{Growth pattern in the recurrent esophageal tumor model}

The primary (0 Gy pre-irradiation) and recurrent (10 Gy or 20 Gy pre-irradiation) esophageal tumor models were established successfully with tumor formation rate of $95 \%$. The TBE was clearly seen through comparison of tumors growth pattern between 0 Gy pre-irradiation and 10 or 20 Gy pre-irradiation groups (Figure 1). Tumors from pre-irradiated mice grew significantly slower than those in the un-irradiated group $(P=0.005$ for 10 Gy $v s 0$ Gy preirradiation, $P<0.001$ for 20 Gy vs 0 Gy pre-irradiation). As for exposure dose, growth delay was much more significant in mice with 20 Gy pre-radiation than those with 10 Gy $(P=0.002)$. The time intervals needed for tumor grown to a $10 \mathrm{~mm}$ average diameter were 17 days and 33 days for 0 Gy and 10 Gy pre-irradiated groups, respectively. Tumors in mice receiving 20 Gy pre-irradiation failed in growing large enough to calculate the above time interval. Therefore, the 10 Gy pre-irradiation group was selected for further radiosensetizing experiments.

\section{Hypoxic characteristics in the recurrent esophageal tumor model}

The characteristics of hypoxia (pimonidazole), tumor blood perfusion (Hoechst 33342), microvessel formation (CD34) and morphology [hematoxylin-eosin (HE)] of tumors were examined and compared (Figures 2 and 3 ) in 0 Gy, 10 Gy and 20 Gy pre-irradiated mice. Necrosis was clearly seen in tumors central area with HE staining. The necrotic fractions were $11.78 \% \pm 2.99 \%$ in 0 Gy pre-irradiation group, while increased significantly to $32.78 \% \pm 7.27 \%$ in 10 Gy pre-irradiated tumors, and $55.52 \% \pm 14.28 \%$ in 20 Gy pre-irradiated tumors, $P<0.001$. Specific staining of capillary-like vessels by antiCD34 was shown in tumor tissues. Pre-irradiation resulted in significant decrease of intra-tumoral microvessel density (MVD) compared with the control. The MVD were $36.67 \pm 5.61$ vessels/field, $25.50 \pm 4.55$ vessels/field, and $11.96 \pm 2.83$ vessels/field in $0 \mathrm{~Gy}, 10 \mathrm{~Gy}$ and $20 \mathrm{~Gy}$ pre-irradiation models, respectively $(P=0.016)$. Analysis of all tumors showed the MVD in tumor was negatively correlated with the necrotic fractions $(\mathrm{r}=-0.751, P=0.005)$.

Pimonidazole binding was primarily confined to peri-necrotic regions while Hoechst 33342 binding located at outer edge of tumor. There was a tendency for intratumoral regions staining positive for pimonidazole or for Hoechst 33342 to be mutually exclusive. The pimonidazole-positive fraction (PPF) and Hoechstpositive fraction (HPF) were calculated for each group. A significant higher PPF and lower HPF were seen in tumors of 20 Gy and 10 Gy pre-irradiation models than 0 Gy pre-irradiation models (PPF: 46.55\% $\pm 15.80 \%, 22.68 \%$ $\pm 7.80 \%$ and $4.92 \% \pm 1.69 \%, P=0.010$; HPF: $38.98 \%$ $\pm 11.79 \%, 59.65 \% \pm 17.74 \%$ and $78.96 \% \pm 23.53 \%$, $\mathrm{P}=0.018$. There were statistical differences between $20 \mathrm{~Gy}$ and 10 Gy pre-irradiation groups as well, which illustrated that the extent of hypoxia and blood perfusion was also dose-dependent.

\section{Radiosensitizing effect of CMNa in the primary and recurrent tumor models}

As shown in Figure 4, irradiation alone inhibited tumor growth in both the primary $(P<0.001)$ and recurrent tumor models $(P<0.001)$. There was no significant difference in growth between radiation alone and $\mathrm{CMNa}$ + radiation $(P=0.285)$ in the primary esophageal xenograft tumors. In contrast, for tumors growing in the recurrent tumor models, the growth rate was remarkably reduced under $\mathrm{CMNa}+$ radiation as comparison with the radiation alone $(P=0.032)$.

The tumor inhibition rate of $\mathrm{CMNa}+$ radiation in the recurrent tumor model was $40.81 \%$ as peak value at 30 days after last fractionation of radiation. However, the maximal inhibition rate of by $\mathrm{CMNa}+$ radiation was only $8.05 \%$ at 35 days after treatments in the primary tumor model. The relative growth delay due to the $\mathrm{CMNa}+$ radiation treatment was much longer than radiation alone in the recurrent tumor model $(P<0.001)$. The relative growth delay times were $7.46 \pm 0.58,28.34 \pm 4.41$, and $35.48 \pm 4.56$ days for blank treatment, radiation alone and 
$\mathrm{CMNa}+$ radiation groups in the primary tumor model. However, the relative growth delay times were 28.52 \pm 3.41 and $85.72 \pm 8.06$ days for blank treatment and radiation alone in the recurrent tumor models. For the $\mathrm{CMNa}+$ radiation tumors in the recurrent tumor models, tumors grown to 2.72 times of initial volume at 97 days after last treatment when observation was ended.

\section{DISCUSSION}

High hypoxic fraction was often seen in recurrent tumors with subsequent poor radiosensitivity. The hypoxic microenvironment and radiosensitizing role of $\mathrm{CMNa}$ in pre-clinical models of recurrent primary tumors were studied in the present work by using ECA109 human esophageal xenografts growing in un-irradiated and pre-irradiated beds in BALB/c-nu/nu mice. Our in vivo experimental exploration succeeded in establishing recurrent esophageal xenograft tumor model, which characterized with significant hypoxic microenvironment.
More importantly, promising radioenhancing roles of $\mathrm{CMNa}$ in established recurrent tumor model was demonstrated and provided theoretical basis for application of $\mathrm{CMNa}$ for recurrent tumors in further clinical trials.

Tumor cells transplanted in pre-irradiated beds of nude mice are frequently used as experimental models of recurrent primary tumors in humans [5-8]. The TBE is considered to be mainly caused by radiation induced injury to the host vasculature and resulted impaired neovascularization. The pre-irradiation dose needed to cause TBE varies according to different tumor types. In our study, 10 Gy and 20 Gy pre-irradiation to tumor beds resulted increased hypoxic fraction, increased necrotic fraction and decreased microvascular density. The hypoxic microenvironment is associated with decreased radiosensitivity and thus remarkable radiosensitizing effect by hypoxic sensitizer. Chen et al [5] reported similar TBE and resistance to re-irradiation and antiangiogenic therapy of sunitinib by implanting mouse prostate $\mathrm{C} 1$ tumors to un-irradiated or pre-irradiated tissues and examining

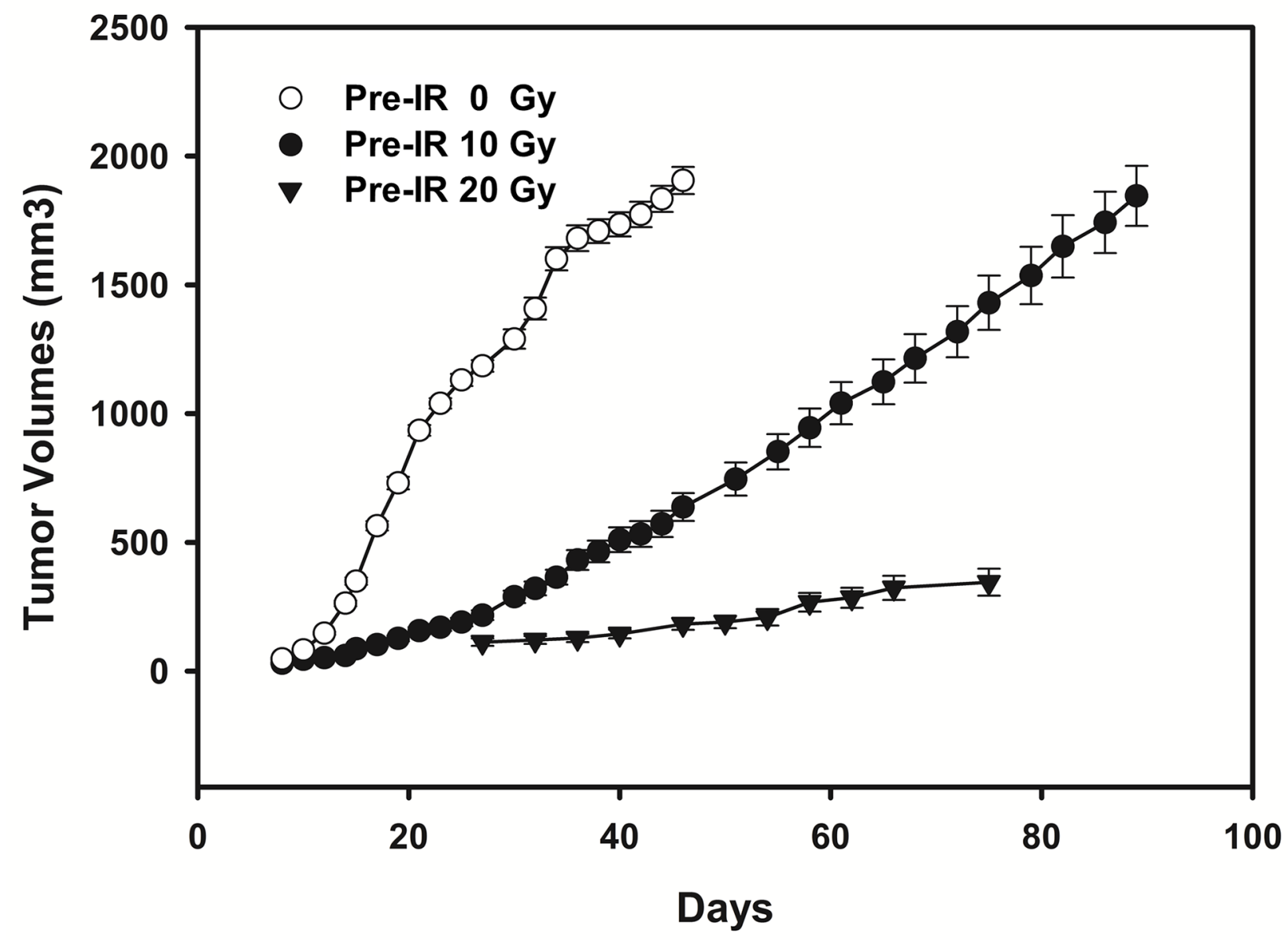

Figure 1: Tumor growth curves of mice ( $n=10$ /group) inoculated with ECA109 cells 24 hours after the 0 Gy, $10 \mathrm{~Gy}$ and $20 \mathrm{~Gy}$ pre-irradiation to the right hind leg. Tumors from $10 \mathrm{~Gy}$ and $20 \mathrm{~Gy}$ pre-irradiated mice grew significantly slower than those in 0 Gy pre-irradiated group ( $P=0.005$ for $10 \mathrm{~Gy} v s 0$ Gy pre-irradiation; $P<0.001$ for 20 Gy $v s 0$ Gy pre-irradiation). As for exposure dose, growth delay was much more significant in mice with 20 Gy pre-radiation than those with 10 Gy $(P=0.002)$. 
vascularity and hypoxia by immunohistochemistry. Additionally, recent researches $[7,21,22]$ had shown that tumor hypoxia may promote metastasis through multiple signal pathways. Rofstad et al [7] showed that preirradiation generated environmental hypoxia is associated with enhanced tumor invasion and metastasis which leaded by transcriptional activation of metastasis-promoting genes including the receptor for the urokinase-type plasminogen activator receptor. In Rezaeian's study [21], the hypoxiaresponsive TRAF6 overexpression promotes breast cancer progression and metastasis to lung and spinal bone, and targeting of TRAF6 reduces breast cancer metastasis, opening up opportunities for therapeutic intervention. Mao et al [22] shows that in HCC cells, hypoxia elevates expression of Cav1, which then acts through the calciumbinding protein S100P to promote metastasis. Further

\section{Pre-IR O Gy}

\section{Pre-IR $10 \mathrm{~Gy}$}
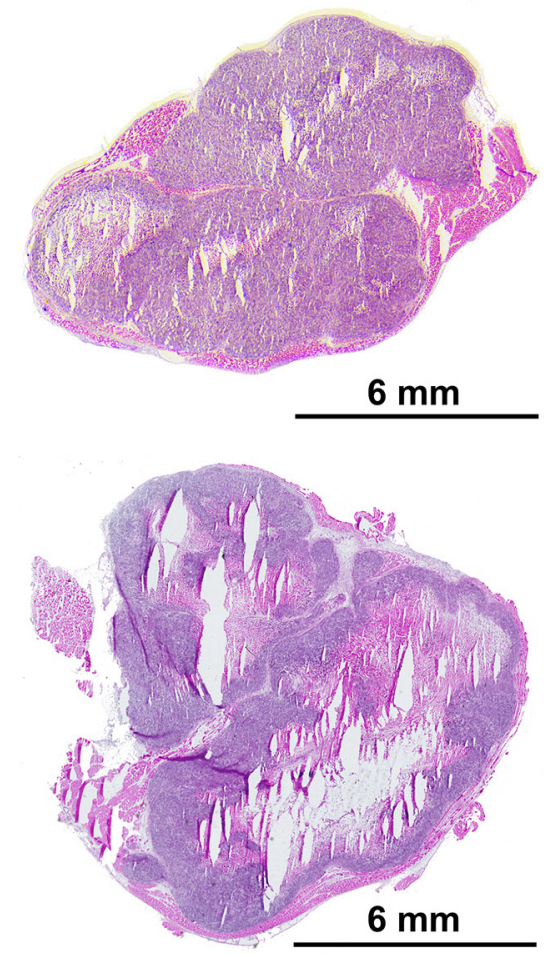

\section{Pre-IR 20 Gy}

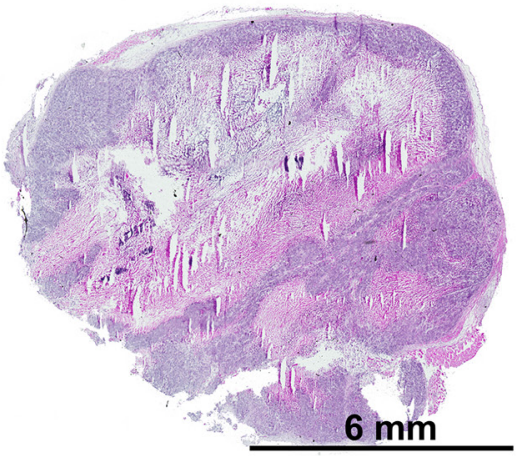

\section{34}
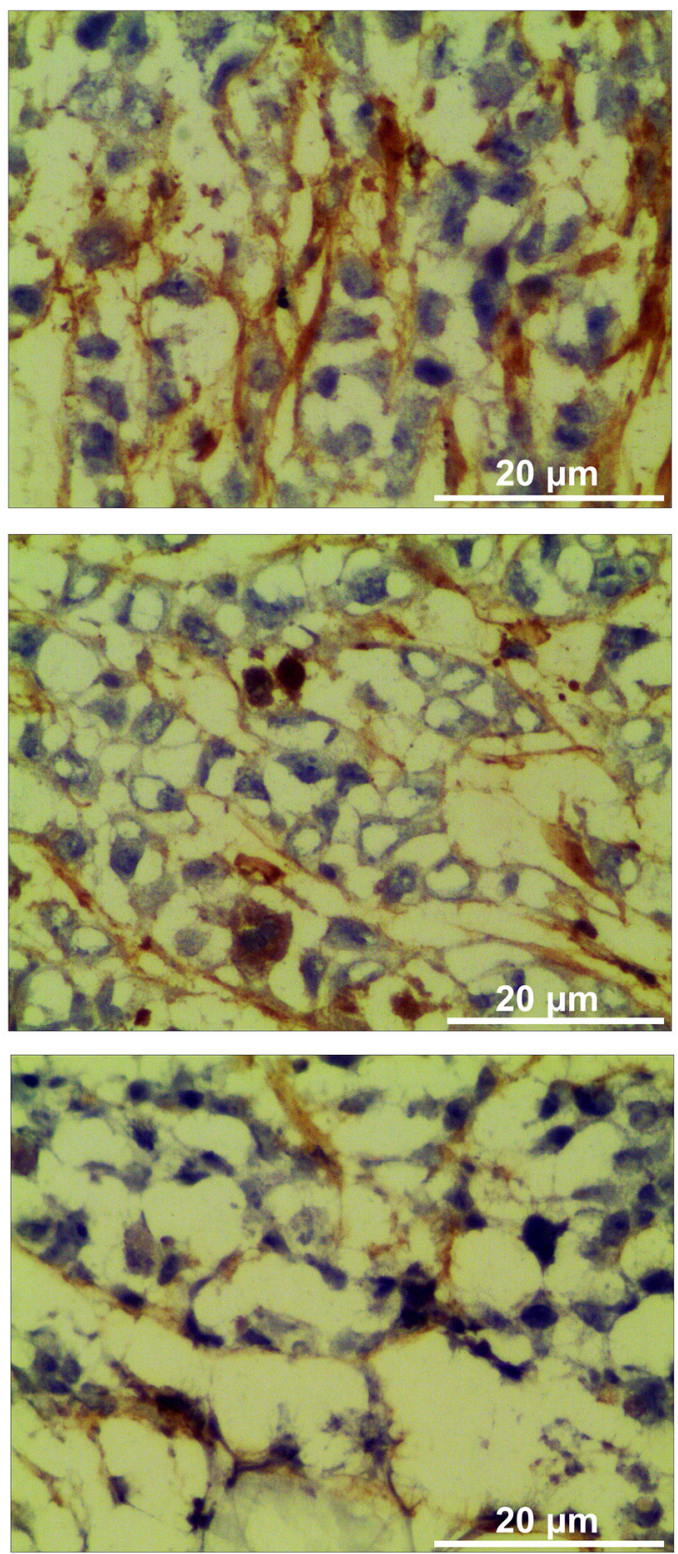

Figure 2: HE staining and CD34 immunohistochemical staining of tumors in the $0 \mathrm{~Gy}, 10 \mathrm{~Gy}$ and $20 \mathrm{~Gy}$ pre-irradiation models. The necrotic fractions derived from HE staining increased as pre-irradiation dose increasing $(P<0.001)$. Specific staining of capillary-like vessels by anti-CD34 showed significant decrease of intra-tumoral microvessel density (MVD) in 10 Gy and 20 Gy pre-irradiation compared with 0 Gy pre-irradiation $(P=0.016)$. MVD in tumor was negatively correlated with the necrotic fractions $(\mathrm{r}=-$ $0.751, P=0.005)$. 
researches about the microenvironment of these recurrent tumor models will be helpful to developing targeted therapy in addition to hypoxia radiosensitizers.

The nitroimidazoles, the most commonly studied hypoxic radiosensitizer, that sensitize radioresistant hypoxic cells to ionizing radiation are believed to mimic oxygen. Oxygen radically alters the pattern of base damage and significantly enhances the level of strand cleavage, especially strand breaks with phosphoglycolate termini. The presence of nitroimidazoles under anoxic conditions does not increase the level of strand breakage than in aerobic conditions, but, like oxygen, significantly enhances the formation of 3'-phosphoglycolate end groups [23]. At a clinically acceptable toxicity level, an expected oxygen enhancement ratio of, at the most, 1.5 to 2.0 can be theoretically achieved by nitroimidazoles [24]. CMNa belongs to 5-nitroimizazole derivatives, which increases the sensitivity of hypoxic tumor cells to radiotherapy by settling down the molecular damage to DNA and preventing potentially lethal damage repair and sublethal damage repair of DNA molecule with less severe toxicity [25]. CMNa has been clinically applied for radiosensitization with improving treatment response without additional adverse events in head and neck

\section{Hoechst 33342 Pimonidazole Fusion}
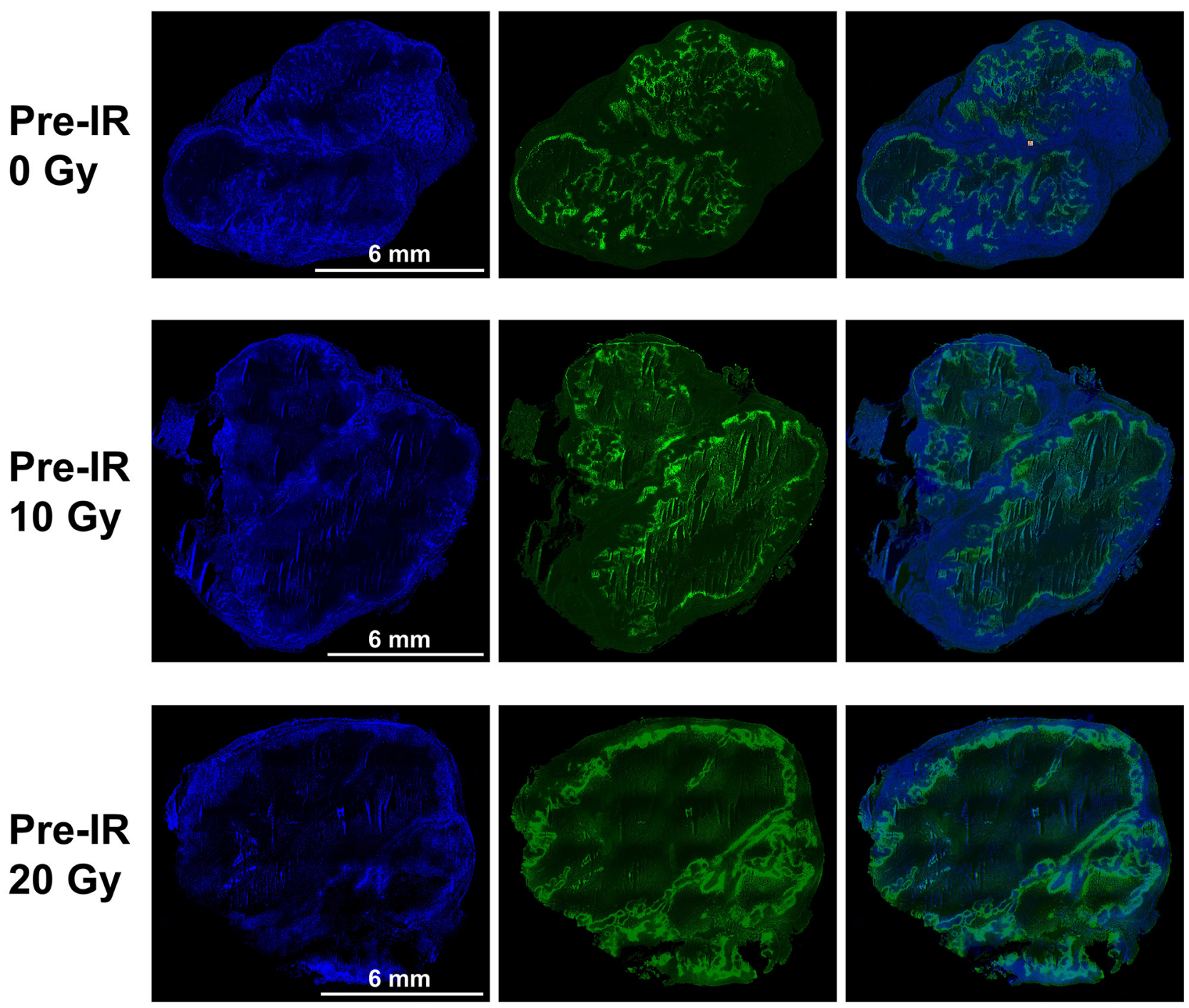

Figure 3: Pimonidazole, Hoechst 33342 and fusion images of tumors in the 0 Gy, 10 Gy and 20 Gy pre-irradiation models. Pimonidazole binding was primarily confined to peri-necrotic regions while Hoechst 33342 binding located at outer edge of tumor. There was a tendency for intratumoral regions staining positive for pimonidazole or for Hoechst 33342 to be mutually exclusive. A significant higher pimonidazole-positive fraction and lower Hoechst-positive fraction were seen in tumors of 10 Gy and 20 Gy preirradiation models than 0 Gy pre-irradiation models $(P=0.010, P=0.018)$. 

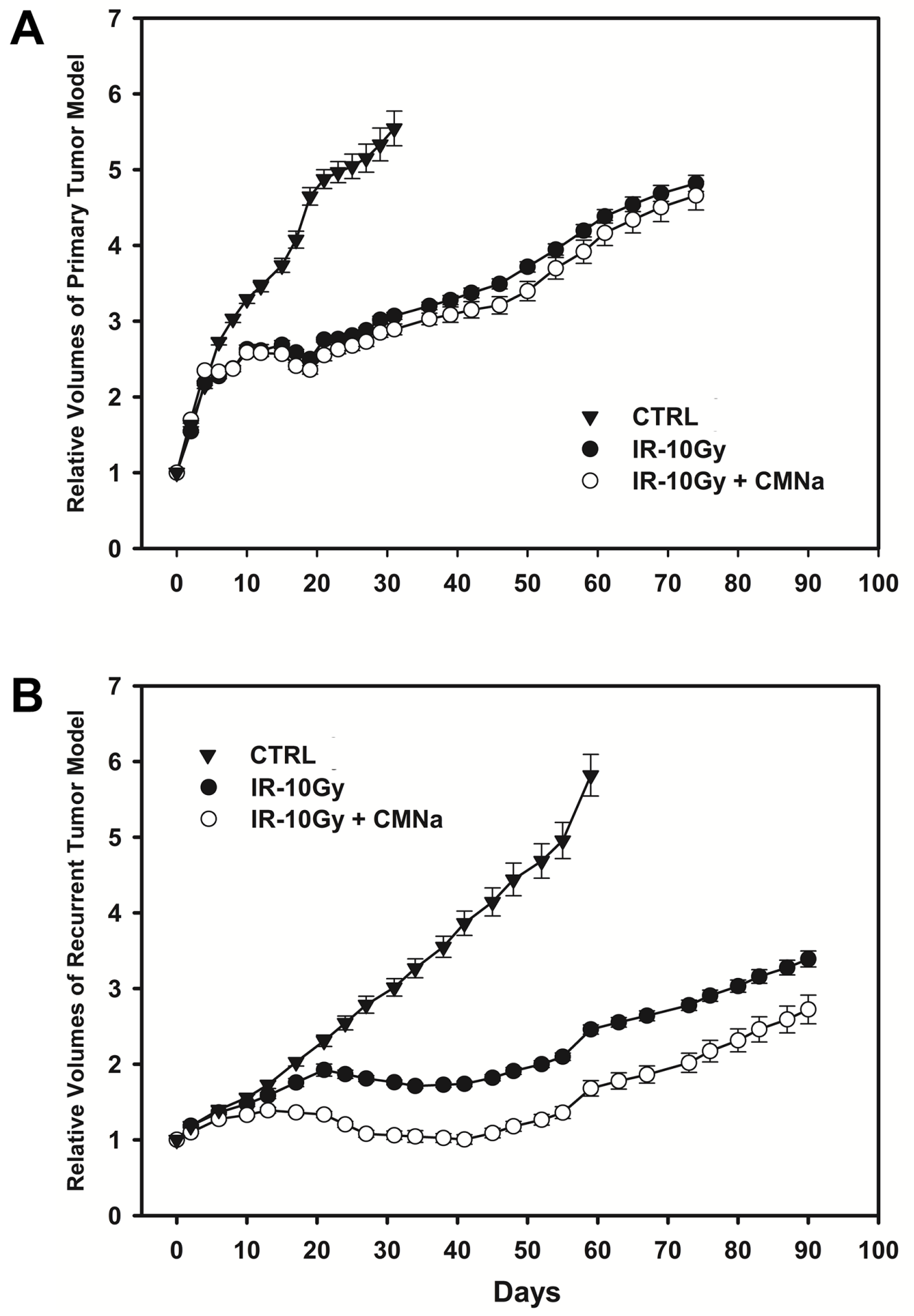

Figure 4: Tumor growth curves of mice with no treatment, irradiation-alone and CMNa+irradiation in the primary and the recurrent esophageal tumor models. (A) Tumor growth curves of mice with no treatment, radiation alone and CMNa + radiation in the primary esophageal tumor model. Radiation alone inhibited tumor growth $(P<0.001)$. There was no significant difference in growth between the radiation alone and $\mathrm{CMNa}+$ radiation $(P=0.285)$. (B) Tumor growth curves of mice with no treatment, radiationalone and $\mathrm{CMNa}+$ radiation in the recurrent esophageal tumor model. Radiation alone also inhibited tumor growth $(P<0.001)$. The growth rate was remarkably reduced under $\mathrm{CMNa}+$ radiation as comparison with the radiation alone $(P=0.032)$ for tumors growing in the preirradiation group. 
cancer and esophageal cancer. Zeng et al [11] compared radiotherapy with and without $\mathrm{CMNa}$ in locally advanced laryngeal squamous cell carcinoma. Overall response rate was significantly improved from $58.33 \%$ to $88.89 \%$. Similarly, He et al [12] reported that radiosensitivity of $\mathrm{CMNa}$ in locally advanced nasopharyngeal carcinoma receiving concurrent radiotherapy and cisplatin-based chemotherapy. Higher complete response rate of $93.33 \%$ were obtained in the CMNa group compared with $73.33 \%$ in the radio-chemotherapy controlled group. Additionally, in NSCLC patients with multiple brain metastases, $\mathrm{CMNa}$ also played effective radiation-enhancing roles, resulted in improved CNS disease control rate $(90.6 \%$ vs $65.6 \%$, $P=0.016$ ), extended median CNS progression-free survival time (7.0 months vs 4.0 months, $P=0.038$ ), and well toleration [19].

$\mathrm{CMNa}$ also been reported to be effect in recurrent tumors. Cheng et al [26] reported 46 patients with recurrent esophageal cancer after complete response to radical radiotherapy. The short-term response rates were $74 \%$ versus $44 \%$ in the $\mathrm{CMNa}$ combination group and re-radiation alone group with statistical significance. The 1-, 2- and 3-year local control rates were 65\%, 39\%, 30\% in the $\mathrm{CMNa}$ combination group and $44 \%, 17 \%, 9 \%$ in the re-irradiation alone group $(P<0.05)$. Liang et al [27] reported similar radio-enhancing effects of $\mathrm{CMNa}$ in locally recurrent nasopharyngeal carcinoma patients with significantly improved local tumor regression rate and equally toxicities. Well-designed large scale multicenter clinical trials on improving therapeutic efficacy of recurrent tumors were still warranted.

Limitations existed in the present study. Firstly, hypo-fractionated radiotherapy with 30 Gy in 3 fractionations at $10 \mathrm{~Gy} /$ fraction were applied and offered good response in radiation-alone group. Secondly, only one esophageal carcinoma cell line of ECA109 was applied. Additionally, single $\mathrm{CMNa}$ concentration $1 \mathrm{mmol} / \mathrm{kg}$ was used which has been verified previously [28]. Future studies would be necessary to be launched to improve the recurrent esophageal tumor model, taking into account the radiotherapy protocols that are used in patients with esophageal cancer. Conventional fractionation schedule instead of hypo-fractionation should be applied during treatment in xenograft tumor nude mice models. Additional esophageal squamous carcinoma cell lines including TE-1 and KYSE30 may be cultured to repeat the experiment protocol to validate the preliminary results. Moreover, pharmacokinetic characteristics and tissue distribution of $\mathrm{CMNa}$ after intravenous administration were explored in mice xenografts models of ECA109, $\mathrm{FaDu}$ and $\mathrm{A} 549$ by investigators from our study group (the article has been accepted but not published online). As a reference, different $\mathrm{CMNa}$ concentrations may be administrated in future protocols.

In conclusion, the present preclinical study demonstrated that the human esophageal xenograft recurrence tumor model with tumor bed effect could be established by a single dose of 10 Gy or 20 Gy preirradiation before tumor transplantation. After preirradiation, the tumors grew much slower with increased hypoxia fraction, decreased blood flow, and decreased radiation sensitivity. Radiosensitization with $\mathrm{CMNa}$ delayed tumor growth significantly. It is suggested that hypoxia radiosensitizer as $\mathrm{CMNa}$ might improve tumor control for patients with in-field recurrences after primary radiotherapy.

\section{MATERIALS AND METHODS}

\section{Cell culture}

The human esophageal squamous carcinoma cell line (ECA109) was purchased from Chinese Academy of Sciences Shanghai Institute of Cell Bank. The cells were cultured in RPMI 1640 medium (Gibco, USA) supplemented with 10\% fetal bovine serum (Gibco, USA), penicillin (100 units $/ \mathrm{ml})$, and streptomycin $(100 \mu \mathrm{g} / \mathrm{ml})$.

\section{Establishment of recurrent tumor model}

The animal experimental protocols has been approved by the institutional research ethics committee of Shandong Cancer Hospital and Institute within which the work was undertaken and that it conforms to the provisions of the Declaration of Helsinki in 1995 (as revised in Edinburgh 2000). All experiments were performed using 6- to 8-week-old female athymic BALB/c nude mice purchased from Beijing Hua Fukang pathogenfree animal breeding facility (approval no. SCXK [Jing] 2009-0008). Nude mice were kept in accordance with institutional guidelines in specific pathogen free units. Tumors were initiated by injecting $5 \times 10^{6}$ cells per mouse in $100 \mu \mathrm{L}$ PBS medium s.c. in the right hind leg of mice.

Nude mice were randomly divided into 0 Gy (unirradiation group), 10 Gy and 20 Gy pre-irradiation groups. Mice were fixed with the right hind leg irradiated (0, 10 or $20 \mathrm{~Gy}$ ) using an X-ray unit (X-Rad 225, PXI, USA) at $225 \mathrm{kV}$ and $13.30 \mathrm{~mA} 24$ hours before tumor transplantation. Mice were inspected daily and tumors measured with a caliper twice per week. Tumor volume was calculated as $\pi / 6 \times a \times b^{2}$, where " $a$ " is the longest and " $b$ " is the shorter of two orthogonal diameters.

\section{Tumor sample preparation and Immunohistochemistry staining}

Six mice in each group were sacrificed when tumor grew to approximately $10 \mathrm{~mm}$ in diameter. The hypoxic cell marker pimonidazole hydrochloride $(16 \mathrm{mg} / \mathrm{mL}$ in saline; $80 \mathrm{mg} / \mathrm{kg}$; Chemicon International) was i.p. injected $2 \mathrm{~h}$ before sacrifice. The Hoechst 33342 (5 mg/ $\mathrm{mL}$ in saline; $25 \mathrm{mg} / \mathrm{kg}$; Sigma-Aldrich) was injected via 
tail vein $1 \mathrm{~min}$ before sacrifice. Immediately after animal sacrifice, tumors were removed quickly and embedded into optimal cutting medium (OCT 4583, Sakura Finetek), and frozen on dry ice. Sets of 10 contiguous $8 \mu \mathrm{m}$ thick tissue sections were prepared for further analysis.

Frozen slides were air dried, fixed in ice-cold acetone for $20 \mathrm{~min}$, and incubated with SuperBlock (Pierce Biotechnology) at room temperature for $30 \mathrm{~min}$. Sections were then incubated with FITC-conjugated anti-pimonidazole monoclonal antibody (Chemicon International) diluted 1:50 in blocking solution for $1 \mathrm{~h}$ at room temperature. Images were acquired at using Nikon H600L ECLIPSE 90i fluorescence microscope (Nikon, Japan) equipped with a motorized stage, NIS-Elements and Image J software. Hoechst 33342 and pimonidazole were imaged using blue and green filters respectively.

Adjacent continuous tumor sections were used for standard HE staining and CD34 immunohistochemistry and imaged by light microscopy. Frozen sections were stained with primary anti-CD34 antibodies (Abcam, Cambridge, USA), washed, and then a brown precipitate was developed at sites of primary antibody binding through use of a peroxidase-conjugated second step antibody and a 3,3-diaminobenzidine reagent.

\section{Immunohistochemistry image analysis}

The images obtained were visually scored blinded by two independent researchers (Jing Liu and Peipei $\mathrm{Wu}$ ) with good inter-observer reproducibility. Images with pimonidazole and Hoechst staining were manually matched to the HE staining images based on the position of tissue edges and necrotic areas by Adobe Photoshop (Adobe, San Jose, CA). Tumor area was defined by contouring the tumor boundary using the HE staining image. Necrotic areas were subtracted from the total area to yield the viable tumor area. Necrosis fraction was defined as the ratio of necrotic area to total area. To generate estimates of the fraction of the tumor area positive for each marker, binary images were created by imposing thresholds. The pimonidazole/Hoechst-positive area was taken to be the number of pixels that had green/ blue fluorescence intensity greater than a threshold value. The PPF was then defined as the ratio of pimonidazolepositive area to viable tumor area. Similarly, the HPF was then defined as the ratio of Hoechst-positive area to viable tumor area. MVD of tumors was evaluated by counting the number of microvessels staining with CD34 per highpower field $(200 \times)$ in the sections [29].

\section{Radiosensitizing effect of CMNa}

Primary (0 Gy pre-irradiation) and recurrent esophageal tumor models (10 Gy pre-irradiation) were established and tumor volumes were monitored as above description. Mice bearing 150-200 $\mathrm{mm}^{3}$ tumors were randomized into 3 groups ( $\mathrm{n}=10$ in each group): A; control (no treatment), B; radiation alone (30 Gy in 3 weekly fractionations), C; radiation (30 Gy in 3 weekly fractionations) combined with $\mathrm{CMNa}(1 \mathrm{mmol} / \mathrm{kg}$ i.p. injected $60 \mathrm{~min}$ before irradiation). Tumor volumes were monitored and compared among groups. Relative tumor volume was defined as the ratio of measured tumor volume at each time point to the initial tumor volume before treatment.

The radiosensitizing effects was quantified by two parameters: (1) the tumor inhibition rate, which was defined as the difference between tumor volumes of radiation alone group and $\mathrm{CMNa}+$ radiation group divide those of radiation alone, and (2) the relative growth delay time, which was defined as the time intervals (days) needed to triple as tumor volumes at the beginning of treatments.

\section{Statistical analysis}

Statistical analysis was performed using SPSS (Version 19.0). Data was expressed as mean values and standard deviations. Analysis of variance (ANOVA) and nonparametric tests were used to compare the tumor volume, necrosis fraction, PPF, HPF and growth delay. The comparison of multiple mean with ANOVA, after the equal check of variance, and the two-two comparisons among the means were done by LSD method. $P<0.05$ was considered statistically significant.

\section{Abbreviations}

$\mathrm{TBE}=$ tumor bed effect, $\mathrm{CMNa}=$ sodium glycididazole, $\mathrm{HE}=$ hematoxylin-eosin, $\mathrm{PPF}=$ pimonidazole-positive fraction, $\mathrm{HPF}=$ Hoechst-positive fraction, $\mathrm{MVD}=$ microvessel density, $\mathrm{ANOVA}=$ analysis of variance.

\section{Author contributions}

Peipei $\mathrm{Wu}$ and Jing Liu performed animal experiment, data analysis, generated figures and drafted the manuscript. Xiaorong Sun participated in the design of the study and in the data evaluation. Xiaolin Li participated in the data analysis and revised the manuscript. Ligang Xing designed the study and revised the manuscript. Jinming Yu participated in the designation of the study. All authors read and approved the final manuscript.

\section{CONFLICTS OF INTEREST}

The authors declare no conflicts of interest.

\section{FUNDING}

This work was supported by The National Nature Science Foundation of China (81572970) and Shandong 
Provincial Natural Science Foundation (ZR2015HZ004, ZR2014YL033).

\section{REFERENCES}

1. Cooper JS, Guo MD, Herskovic A, Macdonald JS, Martenson JA Jr, Al-Sarraf M, Byhardt R, Russell AH, Beitler JJ, Spencer S, Asbell SO, Graham MV, Leichman LL. Chemoradiotherapy of locally advanced esophageal cancer: long-term follow-up of a prospective randomized trial (RTOG 85-01). Radiation Therapy Oncology Group. JAMA. 1999;281:1623-7.

2. Koshy M, Esiashvilli N, Landry JC, Thomas CR Jr, Matthews RH. Multiple management modalities in esophageal cancer: combined modality management approaches. Oncologist. 2004;9:147-59.

3. Zhang X, Li M, Meng X, Kong L, Zhang Y, Wei G, Zhang X, Shi F, Hu M, Zhang G, Yu J. Involved-field irradiation in definitive chemoradiotherapy for locally advanced esophageal squamous cell carcinoma. Radiat Oncol. 2014;9:64.

4. Welsh J, Settle SH, Amini A, Xiao L, Suzuki A, Hayashi Y, Hofstetter W, Komaki R, Liao Z, Ajani JA. Failure patterns in patients with esophageal cancer treated with definitive chemoradiation. Cancer. 2012;118:2632-40.

5. Chen FH, Chiang CS, Wang CC, Fu SY, Tsai CS, Jung SM, Wen CJ, Lee CC, Hong JH. Vasculatures in tumors growing from preirradiated tissues: formed by vasculogenesis and resistant to radiation and antiangiogenic therapy. Int $\mathrm{J}$ Radiat Oncol Biol Phys. 2011;80:1512-21.

6. Chen JJ, Fu SY, Chiang CS, Hong JH, Yeh CK. A preclinical study to explore vasculature differences between primary and recurrent tumors using ultrasound Doppler imaging. Ultrasound Med Biol. 2013;39:860-9.

7. Rofstad EK, Mathiesen B, Henriksen K, Kindem K, Galappathi K. The tumor bed effect: increased metastatic dissemination from hypoxia-induced up-regulation of metastasis-promoting gene products. Cancer Res. 2005;65:2387-96.

8. Kozin SV, Winkler F, Garkavtsev I, Hicklin DJ, Jain RK, Boucher Y. Human tumor xenografts recurring after radiotherapy are more sensitive to anti-vascular endothelial growth factor receptor-2 treatment than treatment-naive tumors. Cancer Res. 2007;67:5076-82.

9. Milas L, Ito H, Hunter N, Jones S, Peters LJ. Retardation of tumor growth in mice caused by radiation-induced injury of tumor bed stroma: dependency on tumor type. Cancer Res. 1986;46:723-7.

10. Kao J, Garofalo MC, Milano MT, Chmura SJ, Citron JR, Haraf DJ. Reirradiation of recurrent and second primary head and neck malignancies: a comprehensive review. Cancer Treat Rev. 2003;29:21-30.

11. Zeng YC, Wu R, Xu ZG, Zhang XY, Fan GL, Wu LN, Wang YM, Hao SH, Zheng W, Chen XD, Chi F, Zhang ZY, Li $\mathrm{X}$, et al. Safety and radiation-enhancing effect of sodium glycididazole in locoregionally advanced laryngeal cancers previously treated with platinum-containing chemotherapy regimens: a preliminary report. Cancer Radiother. 2010;14:59-64.

12. He ZY, Li FY, Tong Q, Liao ZW, Guan XX, Wang Y. [Concurrent chemoradiotherapy with sodium glycididazole and cisplatin for local advanced nasopharyngeal carcinoma]. [Article in Chinese]. Nan Fang Yi Ke Da Xue Xue Bao. 2008;28:2038-40.

13. Gao HJ, Shen G, Wang HM, Yang WW, Su H, Zhai HY, Bao YH. [Phase I clinical trial of sodium glycididazole $(\mathrm{CMNa})$ in cancer patients]. [Article in Chinese]. Zhong Guo Xin Yao Za Zhi. 2004;13:260-3.

14. Fu LQ, Huang F, Guo JH, Gao HZ, Liang YQ, Li J, Wu DZ. [Phase I clinical pharmacokinetics of glycididazolum natrium]. [Article in Chinese]. Zhong Guo Yao Li Xue Yu Du Li Xue Za Zhi. 2004;18:87-92.

15. Li MY, Liu JQ, Chen DP, Qi B, Liang YY, Yin WJ. Glycididazole sodium combined with radiochemotherapy for locally advanced nasopharyngeal carcinoma. Asian Pac J Cancer Prev. 2014;15:2641-6.

16. Liu MZ, He LR, Lu TX, Chen YY, Hu YH, Cui NJ, Xu GZ, Gao L, Xiao GL, Zhang SW, Cai Y, Li DM, Chen LH, et al. [Effect of hypoxic radiosensitizer sodium glycididazole on long-term result of radiotherapy for nasopharyngeal carcinoma]. [Article in Chinese]. Zhonghua Zhong Liu Za Zhi. 2006;28:932-7.

17. Cai L, Liu MZ, Gu MF, Liu H, Chen EC, Hu YH, Lin HX, Wang HY, Huang Y, Li QQ, Cui NJ, Rong TH. [Phase I study of CM-Na combined with concurrent radiochemotherapy for advanced esophageal carcinoma]. [Article in Chinese]. Ai Zheng. 2005;24:582-6.

18. Yang J, Liu MZ, Cai L, Hu YH, Liu H, Li QQ, Cui NJ. [Phase II clinical trial of sodium glyci-didazole (CM-Na) combined with concurrent radiochemotherapy for advanced esophageal carcinoma]. [Article in Chinese]. Ai Zheng. 2008;27:622-6.

19. Zeng YC, Wu R, Xing R, Chi F, Wang SL, Chen XD, Xuan Y, Wu LN, Duan QY, Tang MY, Niu N, Sun YN, Fan GL, et al. Radiation-enhancing effect of sodium glycididazole in patients suffering from non-small cell lung cancer with multiple brain metastases: A randomized, placebocontrolled study. Cancer Radiother. 2016;20:187-92.

20. Zeng YC, Xing R, Zeng J, Xue M, Chi F, Xin Y, Fan GL, Wang HM, Duan QY, Sun YN, Niu N, Wu R. Sodium glycididazole enhances the radiosensitivity of laryngeal cancer cells through downregulation of ATM signaling pathway. Tumour Biol. 2016;37:5869-78.

21. Rezaeian AH, Li CF, Wu CY, Zhang X, Delacerda J, You MJ, Han F, Cai Z, Jeong YS, Jin G, Phan L, Chou PC, Lee $\mathrm{MH}$, et al. A hypoxia-responsive TRAF6-ATM-H2AX signalling axis promotes HIF1 $\alpha$ activation, tumorigenesis and metastasis. Nat Cell Biol. 2017;19:38-51. 
22. Mao X, Wong SY, Tse EY, Ko FC, Tey SK, Yeung YS, Man K, Lo RC, Ng IO, Yam JW. Mechanisms through which hypoxia-induced caveolin-1 drives tumorigenesis and metastasis in hepatocellular carcinoma. Cancer Res. 2016;76:7242-53.

23. Buchko GW, Weinfeld M. Influence of nitrogen, oxygen, and nitroimidazole radiosensitizers on DNA damage induced by ionizing radiation. Biochemistry. 1993;32:2186-93.

24. Liu C, Lin Q, Yun Z. Cellular and molecular mechanisms underlying oxygen-dependent radiosensitivity. Radiat Res. 2015;183:487-96.

25. Overgaard J, Hansen HS, Overgaard M, Bastholt L, Berthelsen A, Specht L, Lindeløv B, Jørgensen K. A randomized double-blind phase III study of nimorazole as a hypoxic radiosensitizer of primary radiotherapy in supraglottic larynx and pharynx carcinoma. Results of the Danish Head and Neck Cancer Study (DAHANCA) Protocol 5-85. Radiother Oncol. 1998;46:135-46.
26. Cheng HH, Fu ZC, Lin GS, Lai HB. [Sensitivity enhancement of sodium glycididazole in re-radiotherapy against local recurrent esophageal carcinoma after radical radiotherapy]. [Article in Chinese]. Di Si Jun Yi Da Xue Xue Bao. 2007;28:39-41.

27. Liang R, Shen X, Chen ZH, Yu ZH. [Radiosensitization of CMNa for locally recurrent nasopharyngeal cancer]. [Article in Chinese]. Zhong Liu Yu Fang Yu Zhi Liao. 2008;21:162-4.

28. Chen M, Li SL, Peng ZP, Wang J, Zhang J. [The chronomodulated radiosensitization by $\mathrm{CMNa}$ on nasopharyngeal carcinoma in vivo]. [Article in Chinese]. Shi Jie Ke Ji Yan Jiu Yu Fa Zhan. 2010;32:101-3.

29. Vermeulen PB, Gasparini G, Fox SB, Toi M, Martin L, McCulloch P, Pezzella F, Viale G, Weidner N, Harris AL, Dirix LY. Quantification of angiogenesis in solid human tumours: an international consensus on the methodology and criteria of evaluation. Eur J Cancer. 1996;32A:2474-84. 